\title{
The Optimal Time to Remove Urinary Catheters in Laparoscopic Partial Nephrectomy, Laparoscopic Adrenalectomy or Laparoscopic Nephrectomy
}

\author{
Hong Caimei $^{1}$, Guo Xiaoxia ${ }^{1}$, Lian Huizhao ${ }^{2,}{ }^{*}$, Yang Qi $^{1}$, Ba Longhong ${ }^{1}$, Chao Xinghui ${ }^{1}$ \\ ${ }^{1}$ Department of Urology, The First Affiliated Hospital of Jinan University, Guangzhou, China \\ ${ }^{2}$ The First Affiliated Hospital, Jinan University, Guangzhou, China
}

Email address:

jinwu122@126.com (Lian Huizhao)

${ }^{*}$ Corresponding author

To cite this article:

Hong Caimei, Guo Xiaoxia, Lian Huizhao, Yang Qi, Ba Longhong, Chao Xinghui. The Optimal Time to Remove Urinary Catheters in Laparoscopic Partial Nephrectomy, Laparoscopic Adrenalectomy or Laparoscopic Nephrectomy. American Journal of Nursing Science. Vol. 9, No. 2, 2020, pp. 66-69. doi: 10.11648/j.ajns.20200902.15

Received: January 13, 2020; Accepted: February 20, 2020; Published: March 6, 2020

\begin{abstract}
Objective To explore the best time to remove the indwelling urinary catheters after laparoscopic partial nephrectomy (LPN), laparoscopic adrenalectomy (LA) or laparoscopic nephrectomy (LN) so as to reduce the indwelling time of nontherapeutic catheters and thus reduce postoperative complications and enhance recovery. Methods We included 140 patients who have undergone laparoscopic partial nephrectomy, laparoscopic adrenalectomy or laparoscopic nephrectomy and received indwelling urinary catheters during the operation in the Department of Urology in our hospital from January 2019 to December 2019. The patients were averagely randomized into control group and observation group. The indwelling urinary catheters in the control group were kept for $3 \mathrm{~d}$ and then nurses removed the catheters following the doctor's advice. In the observation group nurses removed the urinary catheters following the removal procedures after assessing the indications of removal of urinary catheters and necessity of keeping the catheters. After operation, the time of keeping the indwelling urinary catheters, first leaving bed, first passage of gas by anus, average hospital stay, occurrence of constipation and score of painful urination were compared between the two groups. Results In the observation group, after operation, the average time of keeping the indwelling urinary catheters is $7.94 \pm 1.54 \mathrm{~h}$, the average time of first leaving bed activity is $18.65 \pm 6.14 \mathrm{~h}$, first passage of gas by anus is $16.18 \pm 2.44 \mathrm{~h}$, and the average hospital stay is $5.71 \pm 1.93 \mathrm{~d}$. Compared with control group, there is a significant difference $(\mathrm{P}<0$. 05). There is also a significant difference in the occurrence of constipation between the two groups with 6 cases in control group but 0 in observation group $\left(\chi^{2}=4.353, \mathrm{P}=0.037\right)$. Patients in both groups have urination discomfort to different degrees and the score of pain is significantly different between the two groups $\left(\chi^{2}=5.079, \mathrm{P}=0.024\right)$. Conclusions The indwelling urinary catheters for intraoperative needs are advised to remove within $12 \mathrm{~h}$ after laparoscopic partial nephrectomy, laparoscopic adrenalectomy or laparoscopic nephrectomy, which can enhance patients' comfort level and recovery, reduce length of stay and hospitalization costs, and save medical costs.
\end{abstract}

Keywords: Laparoscopic Surgery, Indwelling Urinary Catheters, Remove Time

\section{Introduction}

Indwelling urinary catheter is a common operation in surgery which is aimed to avoid accidental injury to bladder and monitor urine volume. After operation, the indwelling urinary catheters have fulfilled their task and should be removed in time. However, due to various reasons, belated removal of catheters is a common case. The indwelling catheters bring discomfort to patients. Research shows the rate of discomfort is up to $73.2 \%$ which brings great inconvenience to patients' daily life [1-3] especially their leaving bed activities in the early period of postoperation. Long stay in bed is likely to cause relative complications, slowing rehabilitation. The "Chinese Expert Consensus and Path Management Guidelines for Enhanced Recovery after Surgery" points out that the catheters should be removed 24 hours after operation 
and leaving bed activity is advised at the first day postoperatively $[4,5]$.

During the operations for urological diseases such as kidney neoplasms, cyst of kidney and disease of adrenal gland, indwelling urinary catheters are regular device for urine drainage, urine monitoring and anesthetic management. For operations for urinary calculus, prostatic hyperplasia and bladder tumor, intraoperative and postoperative indwelling catheters are for keeping low bladder pressure or preventing urinary reflux. However, there is no a single standard clinical and nursing instruction for the time of removal of nontherapeutic indwelling urinary catheters after operation for kidney neoplasms, cyst of kidney and disease of adrenal gland.

Our department has developed the operative treatments such as laparoscopic partial nephrectomy, laparoscopic adrenalectomy, laparoscopic nephrectomy for urological diseases. Regularly, after LPN, LA and LN, the urinary catheters are kept for 2 to $3 \mathrm{~d}$. However, the indwelling catheters bring discomfort to patients and affect their leaving bed activities in the early period of postoperation, causing long stay in bed and slowing postoperative recovery. Hence, we explore the best time to remove the nontherapeutic indwelling urinary catheters after LPN, LA and LN so as to reduce postoperative complications and enhance recovery after surgery. We conducted the control clinical trial from January 2019 to December 2019. During the trial, we found that the indwelling catheters for intraoperative needs can be removed within $12 \mathrm{~h}$ postoperatively, which leads to patients' earlier leaving bed activities and enhances recovery of gastrointestinal function. Therefore, the removal of catheters within 12 hours postoperatively enhances patients' postoperative recovery, reduces hospital stay and hospitalization costs, and saves medical costs. Thus it is worth clinical promotion and application. The trial is reported as follows.

\section{Data and Methods}

\subsection{General Data}

We enrolled 140 patients who were given laparoscopic partial nephrectomy, laparoscopic adrenalectomy or laparoscopic nephrectomy under general anesthesia and had indwelling urinary catheters in the Department of Urinary Surgery from January 2019 to December 2019 and averagely randomized them into control group and observation group. In the control group, there are 41 males and 29 females, aging from 20 to 75, with an average age of 45.8 \pm 3.6 . Among them, there are 18 cases of LPN, 23 cases of LA and 29 cases of LN. In the observation group, 39 are males and 31 females, aging from 22 to 79 , with an average age of $47.3 \pm 6.7$, and there are 16 cases of LPN, 24 cases of LA, and 30 cases of LN. The inclusion criteria are: (1) having received LPN, LN or LA under general anesthesia and without serious complications; (2) no prostatic hyperplasia in male patients in both groups; (3) indwelling urinary catheters for intraoperative needs. There is no significant difference in the general data between two groups $(\mathrm{P}>0.05)$.

\subsection{Methods}

\subsubsection{Removal of Catheters in the Control Group}

Doctors advised to remove the urinary catheters within 3 days postoperatively and nurses follow the doctors' advice.

\subsubsection{Removal of Catheters in the Observation Group}

We made evidence-based urination training guide, assessment sheet for indications of removal, removal procedure guide, recording sheet and supervisory system for removal of urinary catheters. The guides include: (1) the on-duty nurse gave urination training in bed for patients according to the guides until the patients could urinate without difficulty; (2) the on-duty nurse assessed the indications of removal of catheters of the patients according to the assessment sheet 6 hours after the patients were sent back to the wards, and assessed every 2 hours until the catheters were removed; (3) nurses filled the recording sheet for removal of urinary catheters; (4) the charge nurse checked the condition of removal every day and checked the recording sheet for removal at an irregular interval every week. The content of assessment sheet for indications of removal of urinary catheters include: a. clear consciousness; b. stable vital signs; c. no need for precise monitoring of urine volume; $d$. no bladder outlet obstruction (no prostatic hyperplasia under B-ultrasonic examination of urinary system); e. no blood urine; f. less-than-4 pain score or analgesic intervention for over-4 pain score. When all the above indications are met and doctors approve of removal, the catheters can be removed.

\subsection{Outcome Measurements}

(1) The average time of keeping the indwelling urinary catheters of both groups after operation; (2) the time of first leaving bed activity; (3) the time of first passage of gas by anus; (4) constipation (no defecation for three days after operation); (5) length of stay; (6) hospitalization costs; (7) comfort level of urination after removal of catheters which is graded with numerical rating scales (NRS) (0 means no pain; 1-3 means mild pain and no impact on sleep; 4-6 moderate pain and mild impact on sleep; 7-9 severe pain causing that patients cannot fall asleep or wake up from the pains; 10 means sharp pain).

\subsection{Statistical Analysis}

We used SPSS 17.0 for statistical analysis. The measurement data were shown as mean \pm standard deviation and tested by t-test. Enumeration data were tested by $\chi^{2}$ and $\mathrm{p}<0.05$ means significant difference.

\section{Results}

\subsection{Comparison of Outcome Measurements Between Two Groups}

In the observation group, the time of keeping the indwelling 
urinary catheters ranges from 4 to 12 hours, with an average of $7.94 \pm 1.54 \mathrm{~h}$; the time of first leaving bed activity ranges from 10.0 to 29.0 hours, with an average of $18.65 \pm 6.14 \mathrm{~h}$; the time of first passage of gas by anus ranges from 12.0 to 23.0 hours with an average of $16.18 \pm 2.44$ and the length of stay ranges from 2 to $12 \mathrm{~d}$ with an average of $5.71 \pm 1.93 \mathrm{~d}$. In the observation group, the time of keeping the indwelling urinary catheters is from 25 to $26 \mathrm{~h}$ and the average time is $40.4 \pm 7.75$ $\mathrm{h}$; the time of first leaving bed activity from 18.0 to 68.0 hours with an average of $41.24 \pm 10.33 \mathrm{~h}$; the time of first passage of gas by anus from 21.0 to $35.0 \mathrm{~h}$ and average time $28.31 \pm 3.75 \mathrm{~h}$; and the length of stay from 4 to 11 days with an average of $7.26 \pm 1.63 \mathrm{~d}$. There is a significant difference in these four outcome measurements between control group and observation group $(\mathrm{P}<0.05)$. The data are shown in the Table 1. There is also a significant difference in the occurrence of constipation with no occurrence in the observation group and 6 cases in the control group $\left(\chi^{2}=4.353, p=0.037\right)$.

Table 1. Comparison of Outcome Measurements between Two Groups.

\begin{tabular}{|c|c|c|c|c|}
\hline Outcome measurements & Control group & Observation group & $t$ & $\boldsymbol{P}$ \\
\hline Average time of keeping catheters postoperatively (h) & $40.4 \pm 7.75$ & $7.94 \pm 1.54$ & 34.357 & 0.000 \\
\hline Average time of first leaving bed activity postoperatively (h) & $41.24 \pm 10.33$ & $18.65 \pm 6.14$ & 15.721 & 0.000 \\
\hline Time of first passage of gas by anus postoperatively (h) & $28.31 \pm 3.75$ & $16.18 \pm 2.44$ & 22.628 & 0.000 \\
\hline Average length of stay (d) & $7.26 \pm 1.63$ & $5.71 \pm 1.93$ & 5.102 & 0.000 \\
\hline Average hospitalization costs RMB (10 thousand) & $23583.02 \pm 1818.54$ & $20242.79 \pm 1019.78$ & 13.341 & 0.000 \\
\hline
\end{tabular}

\subsection{Comparison of Urination Pain Score After Removal of Catheters Between Two Groups}

Patients in both groups have urination discomfort to different degrees. In the observation group, there are 2 cases of mild pain, 1 case of moderate pain while in the control group, there are 7 cases of mild pain and 4 cases of moderate pain. There is a significant difference in the urination pain score between the two groups $\left(\chi^{2}=5.079, \mathrm{p}=0.024\right)$ which is shown in the Table 2.

Table 2. Comparison of Urination Pain Score after Removal of Catheters between Two Groups.

\begin{tabular}{lllll}
\hline \multirow{2}{*}{ Groups } & \multirow{2}{*}{ N } & Pain & & \\
\cline { 3 - 5 } & & No pain & Mild pain & Moderate pain \\
\hline Control group & 70 & $59(84.3)$ & $7(10.0)$ & $4(5.7)$ \\
Observation group & 70 & $67(95.7)$ & $2(2.9)$ & $1(1.4)$ \\
$\chi^{2}$ & & & 5.079 & \\
$P$ & & & 0.024 & \\
\hline
\end{tabular}

\section{Discussion}

During operations for renal tumor, renal cyst, diseases of adrenal gland and other urological diseases, indwelling urinary catheters for drainage of urine, monitoring urine volume and convenient management of anesthetists is a common procedure. Indwelling urinary catheters for intraoperative needs have fulfilled their tasks and should be removed at the end of the operation. However, patients under anesthesia are unconscious of micturition desire and cannot urinate voluntarily because their sensory and motor functions have not recovered, or the postoperatively vital signs are not stable, so the indwelling urinary catheters are kept and brought to the wards.

Some research show that a longer time of keeping indwelling urinary catheters results in higher incidence of bacteriuria and blood urine and more severe pain in first urination [6]. In USA, among all the infections, incidence of catheter-associated urinary tract infection (CAUTI) is up to $34 \%$ [7-9]. The urinary tract infection takes up $40 \%$ of all that iatrogenic infections and indwelling urinary catheter is a major risk factor of that [10-11]. Therefore, to explore a best time for removal of urinary catheters is very important.

In our department, indwelling catheters received during LPN, LA or LN have been regularly kept for 3 days postoperatively and nurses remove the catheters following doctor's advice. Nurses seldom assess and discuss with doctors the necessity of indwelling catheters for patients. This will cause that the catheters are kept for an unnecessarily longer time. The urinary tract contains many nerves, and the sympathetic and parasympathetic nerves are located throughout the urinary tract, so the indwelling urinary catheters will induce discomfort and pain to different degrees $[12,13]$, especially when patients move. As a result, patients are reluctant or even afraid to leave bed for activities. However, when staying in bed for a long time patients' gastrointestinal function may recover slowly which has a negative impact on their appetite and insufficient supply of nutrition is unfavorable to the healing of wound and recovery of body. Besides, staying in bed for a long time is very likely to lead to pressure sores and deep venous thrombosis which are also unfavorable to patients' recovery. Chinese Expert Consensus and Path Management Guidelines for Enhanced Recovery after Surgery points out earlier leaving bed activity can promote recovery of breath, gastrointestinal system, musculoskeletal system and other systems. Also, it is favorable to the prevention of pulmonary infection, pressure sores and deep venous thrombosis $[6,12]$.

In the current research, we found that the indwelling time of urinary catheters in the observation group was $10.2 \pm 1.8 \mathrm{~h}$, time of first leaving bed activity was $18.0 \pm 5.5 \mathrm{~h}$, time of first passage of gas by anus was $17.0 \pm 5.0 \mathrm{~h}$, and average length of stay was $8 \pm 0.5 \mathrm{~d}$, which were all shorter than those in the control group. Besides, the incidence of postoperative complications and hospitalization costs of observation group were lower than those of control group, and pain on urination was milder than that of control group. There was a significant difference $(\mathrm{P}<0.05)$, which was consistent with other relevant research. Catheters could be removed $23 \mathrm{~h}$ after 
retroperitoneal laparoscopic surgery [14]. In the present research, the longer time of first leaving bed activity, of first passage of gas by anus and slower recovery of gastrointestinal function in the control group are because of longer time of keeping indwelling urinary catheters which brings discomfort to patients. Some patients may be afraid of defecating because the discomfort in urinary tract will be aggravated when trying hard to defecate, which may affect the recovery and prolong length of stay. Sympathetic and parasympathetic nerves that control detrusor of bladder and sphincter vesicae have recovered 4 to $6 \mathrm{~h}$ after operation, and urination has also recovered from anesthetic state. Hence, nurses can assess patients' function of urination $6 \mathrm{~h}$ after operation [15-16]. We found that removal of catheters within $12 \mathrm{~h}$ can enhance comfort level, reduce hospitalization costs, nurses' work and improve nursing efficiency.

\section{Conclusions}

In conclusion, that nurses make assessment sheet and procedure guide for removal of urinary catheters according to Chinese Expert Consensus and Path Management Guidelines for Enhanced Recovery after Surgery can ensure implementation rate and patients' safety and at the same time promote recovery of patients. Despite too small sample size, the current research suggests that indwelling urinary catheters for intraoperative needs can be removed within $12 \mathrm{~h}$ after operation, which can reduce postoperative complications, enhance patients' comfort level and promote their recovery. As a result, removal within $12 \mathrm{~h}$ can reduce length of stay, save medical costs and thus is worth clinical promotion.

\section{References}

[1] Dellimore, K. H., Helyer, A. R., \& Franklin, S. E. (2013). A scoping review of important catheter-induced complications. Journal of Materials Science Materials in Medicine, 24 (8), $1825-1835$.

[2] Ramanathan, R., \& Duane, T. M. (2014). Urinary tract infections in surgical patients. Surgical Clinics of North America, 94 (6), 1351-1368.

[3] Lam, T. B. L., Omar, M. I., Fisher, E., Gillies, K., \& Maclennan, S. (2014). Types of indwelling urethral catheters for short-term catheterisation in hospitalised adults. Cochrane Database Syst Rev, 9 (9), CD004013-CD004013.

[4] Li, L., Chen, J., Liu, Z., Li, Q., \& Shi, Y. (2017). Enhanced recovery program versus traditional care after hepatectomy: a meta-analysis. Medicine, 96 (38), e8052.

[5] Yang, R., Tao, W., Chen, Y. Y., Zhang, B. H., Tang, J. M., \& Zhong, S., et al. (2016). Enhanced recovery after surgery programs versus traditional perioperative care in laparoscopic hepatectomy: a meta-analysis. International Journal of Surgery, $36,274-282$.

[6] Wu, S., Chen, W., Shen, L., Xu, L., Zhu, A., \& Huang, Y. (2017). Risk factors for prolonged hypotension in patients with pheochromocytoma undergoing laparoscopic adrenalectomy: a single-center retrospective study. Scientific Reports, 7 (1), 5897.

[7] Fink, R., Gilmartin, H., Richard, A., Capezuti, E., Boltz, M., \& Wald, H. (2012). Indwelling urinary catheter management and catheter-associated urinary tract infection prevention practices in nurses improving care for healthsystem elders hospitals. American Journal of Infection Control, 40 (8), 715-720.

[8] Rupp, M. E., Fitzgerald, T., Marion, N., Helget, V., Puumala, S., \& Anderson, J. R., et al. (2004). Effect of silver-coated urinary catheters: efficacy, cost-effectiveness, and antimicrobial resistance. American Journal of Infection Control, 32 (8), 445-450.

[9] Olsen-Scribner, R. J., Hayes, C., \& Pottinger, P. (2014). Sustaining reduction of catheter-associated urinary tract infection (cauti) - outcomes after two educational methods in a regional university-affiliated medical center. American Journal of Infection Control, 42 (6), S22.

[10] Sanjay, S., Trautner, B. W., Fowler, K. E., John, C., David, R., \& Erica, L., et al. (2018). A multicenter study of patient-reported infectious and noninfectious complications associated with indwelling urethral catheters. JAMA Internal Medicine, 178 (8), 1078-1085.

[11] Shimoni, Z. (2014). Reducing urinary catheter use in patients hospitalized in internal medicine departments. Harefuah, 153 (7), 411-4.

[12] Mohsen Adib-Hajbaghery, \& Mohammad Aghajani. (2009). Quality of care for patients with indwelling urinary catheter in selected hospitals in kashan, iran 2007. International Journal of Urological Nursing, 3 (2), 43-49.

[13] Agarwal, B. K., \& Agarwal, N. (2016). Randomized controlled trial on the efficacy of bladder training before removing indwelling urinary catheter in patients with acute urinary retention. China Biogas, 61 (2), 219-226.

[14] Laan, B. J., Spijkerman, I. J. B., Godfried, M. H., Pasmooij, B. C., \& Geerlings, S. E. (2017). De-implementation strategy to reduce the inappropriate use of urinary and intravenous catheters: study protocol for the ricat-study. BMC Infectious Diseases, 17 (1), 53.

[15] Kwaan, M. R., Lee, J. T., Rothenberger, D. A., Melton, G. B., \& Madoff, R. D. (2015). Early removal of urinary catheters after rectal surgery is associated with increased urinary retention. Diseases of the Colon \& Rectum, 58 (4), 401-405.

[16] Hu, Y., Craig, S. J., Rowlingson, J. C., Morton, S. P., Thomas, C. J., \& Persinger, M. B., et al. (2014). Early removal of urinary catheter after surgery requiring thoracic epidural: a prospective trial. Journal of Cardiothoracic and Vascular Anesthesia, 28 (5), 1302-1306. 